\title{
Nano-Sensor and Circuit Design for Anti-Cancer Drug Detection
}

\author{
S. Sara Ghoreishizadeh, Camilla Baj-Rossi, Sandro Carrara*, Giovanni De Micheli \\ EPFL - École Polytechnique Fédérale de Lausanne $(\mathrm{CH})$ \\ *corresponding author: sandro.carrara@epfl.ch
}

\begin{abstract}
The development of new technologies to monitor drugs concentration directly in patient's blood is absolutely required to succeed in personalized drug therapies. In this study, Etoposide - a well-known anti-cancer drug - has been chosen as model for cyclic voltammetry detection of drugs. Carbon nanotubes are chosen as electron-transfer mediators to enhance the system sensitivity. A very low frequency and low slope triangular-wave potential is required to acquire cyclic voltammograms. Cyclic voltammograms are definitely needed for a correct identification and quantification of the drug concentration in the patient serum. The aim of the paper is to investigate the feasibility of VLSI fully-integration of cyclic voltammetry measurements as a tool to develop a low-cost chip for drug monitoring in personalized therapy. A triangular wave generator CMOS circuit is proposed by using Direct Digital Synthesis (DDS) method. The circuit is implemented in $0.18 \mu \mathrm{m}$ technology and it presents the possibility of changing the slope of the triangular voltage in a wide range of 10 to $100 \mathrm{mV} / \mathrm{s}$.
\end{abstract}

Index Terms- anti-cancer drug, personalized therapy, carbon nanotube, cyclic voltammetry, low frequency, ramp-generator circuit.

\section{INTRODUCTION}

In these last years it has been recognized that any drug therapy needs to be modified according to the individual patient, leading to the personalization of the pharmacological treatment. It has been reported [1] that the response rate of patients to drugs used in major diseases is typically in the range of $20 \%$ to $50 \%$. There are many reasons why a patient might or might not respond to a drug or be affected by an adverse event. Among these are mis-dosing, drug-drug interactions, drug allergies and medication errors. However the main reason for inappropriate drug response has to be found in the genetic polymorphism of the proteins involved in drug metabolism. The present state-of-the-art technology offers some tools for monitoring the genetic predisposition to drug metabolism, but they only predict the response to the drug administration. Another tool is required to measure the drug concentration in the patient's body during the pharmacological cure, which is not yet offered. These measurements are essential in the case of some pharmacological treatments based on drug regimens, such as anti-cancer drug therapies [2].

Etoposide is a widely used anti-cancer drug, administered for the treatment of many kinds of tumours [3, 4]. In this work a Carbon NanoTube (CNT)-based biosensor for the direct monitoring of Etoposide has been proposed. The CNTs were used to obtain highly sensitive sensor [5]. The biosensing has carried out with the cyclic voltammetry, where the drug concentration is detected and quantified by measuring current peaks. Cyclic voltammetry is a wellknown technique that is widely used in various applications where electrochemical analysis is required. In order to measure the cyclic voltammogram of any drug, a very low frequency triangular voltage is applied between working and reference electrodes of the electrochemical cell and the current through working electrode is plotted versus the applied voltage using laboratory instruments. In recent applications in personalized therapy, which use implantable sensor to measure different drug and metabolism characteristics, the setup to generate the ramp voltage and read out the current has to be integrated on a single chip. Since the period of the ramp is very long (in the range of tens of seconds), it cannot be designed with usual methods. In this work, a technique to generate the appropriate ramp for cyclic voltammetry of a wide range of drugs is presented. In the current measurement side, some works have been done recently and been presented in [6].

In this paper cyclic voltammetry has been applied with different concentrations of Etoposide, and a circuit architecture to measure the cyclic voltammetry has been designed for integrate circuits development with $0.18 \mu \mathrm{m}$ technology.

\section{MATERIALS AND METHODS}

\section{A. Materials}

The electrodes were made of graphite working electrode (area, $12.56 \mathrm{~mm}^{2}$ ), a graphite counter electrode and an $\mathrm{Ag} / \mathrm{AgCl}$ reference electrode. The total area of the cell is $22 \mathrm{~mm}^{2}$. Carbon paste screen-printed electrodes (model DRP110 ) and Multi walled carbon nanotubes (MWCNT) in powder (95\% purity, with $-\mathrm{COOH}$ groups present at $5 \%$ ) were purchased from Dropsens (Spain). The MWCNT average diameter and length are $10 \mathrm{~nm}$ and $1-2 \mu \mathrm{m}$ respectively. MWCNT powder was diluted in Chloroform to The concentration of $1 \mathrm{mg} / \mathrm{ml}$ and then sonicated for 30 minutes to obtain a homogeneous suspension with breaking 


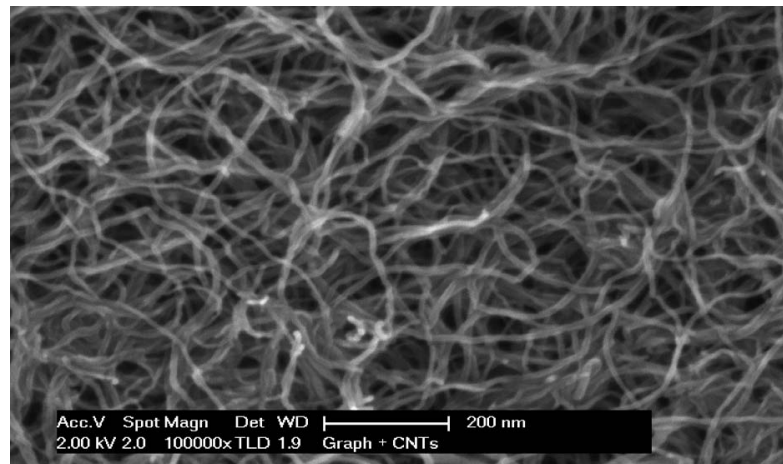

Fig 1. SEM of a central area of a graphite electrode with MWCNTs

macro-aggregates. Etoposide drug, purchased from Sigma in powder, was firstly dissolved in DMSO (dimethyl sulfoxide), and then diluted in Phosphate Buffered Saline (PBS) with $\mathrm{pH}$ 7.4 in aliquots of 5-10-20-30-40-50mM. A Philips/FEI XL$30 \mathrm{~F}$ microscope (with $2.5 \mathrm{~nm}$ resolution in UHR mode at 1 $\mathrm{kV}$ ) has been used to acquire Scanning Electron Microscopic (SEM) images. The high Hydrophobicity of the CNT-surface assures a quite good and stable in time electrical interface between the CNT and the electrodes. Then, the CMOS circuits under design will be connected to the screen-printed and nano-structured electrodes by means of commercially available interfaces for the embedded integration.

\section{B. Electrode nanostructuration}

$30 \mu 1$ of MWCNT-chloroform solution was deposited onto the working electrode by drop casting: after having sonicated the solution of MWCNTs and chloroform for 3-4s to break macro-aggregates, $2 \mu \mathrm{l}$ of the solution were drop cast each time until all $30 \mu$ of the solution was deposited. After the deposition, the chloroform evaporated and the nanotubes laid down the electrode surface forming a 3D porous structure. Fig. 1 shows the acquired picture with SEM for CNTs drop casted onto the electrode surface. As it is shown in this picture, when carbon nanotubes are dropped onto the surface they form agglomerates of thin wrapped wires spread onto the surface.

\section{Electrchemical measurements}

The Etoposide detection was carried out by cyclic voltammetry under aerobic conditions. Voltammograms were

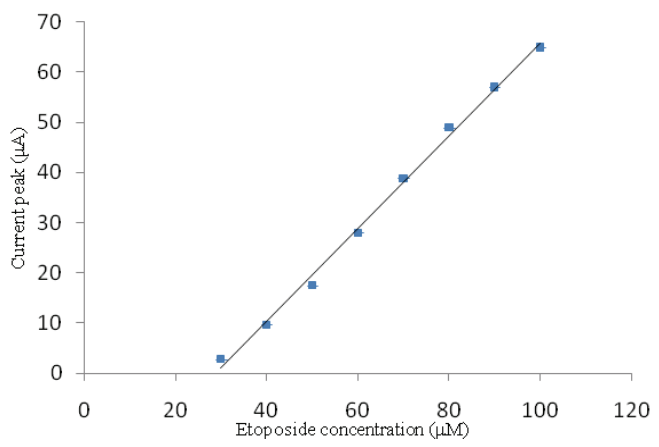

Fig 3. Calibration curve obtained for Etoposide in the concentration range $30 \mu \mathrm{M}-100 \mu \mathrm{M}$

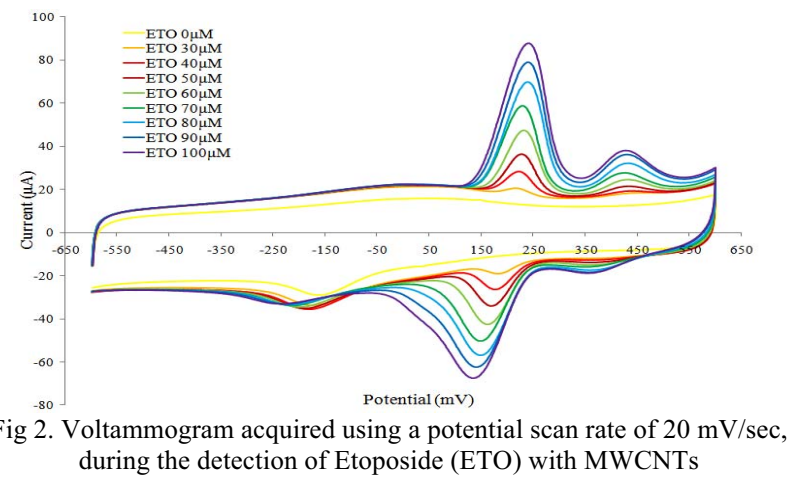

acquired by using a Versastat 3 potentiostat (Princeton Applied Technologies). The electrode was covered with 100 $\mu \mathrm{l}$ of PBS $100 \mathrm{mM}$ (PBS 10X) at $\mathrm{pH} 7.4$ to form the electrochemical cell. To obtain the voltammetric response to different drug concentrations, drops of drug solution with different concentration according to its pharmacological range (30-100 $\mu \mathrm{M}[7])$, were added to the $100 \mu \mathrm{l}$ drop of PBS covering the electrode. The potential of the working electrode swept in the range of -600 to $+600 \mathrm{mV}$ vs $\mathrm{Ag} / \mathrm{AgCl}$ with a scan rate of $20 \mathrm{mV} / \mathrm{sec}$. The peaks obtained in cyclic voltammetry were analyzed, and the values of current peaks at different drug concentrations were calculated using Gaussian approximation [8], to obtain the calibration curve.

\section{EXPERIMENTAL RESULTS}

The voltammetric response to different concentrations of Etoposide was measured. Results of these experiments are reported in Fig. 2 and 3. Fig. 2 reports the Etoposide detection as registered in cyclic voltammograms. It clearly shows that every single peak is growing up upon Etoposide concentration. The peak at around $-200 \mathrm{mV}$ is due to the oxygen moieties derived from Carbon-nanotubes as has been already reported $[9,10]$. Two oxidation peaks at $+220 \mathrm{mV}$ and $+450 \mathrm{mV}$ and a reduction peak at $+150 \mathrm{mV}$ are clearly visible. Another reduction peak at $+50 \mathrm{mV}$ is partially hidden by the peak at $+150 \mathrm{mV}$. These data confirm the peaks reported in [11] obtained through Etoposide cyclic voltammetry at glassy carbon electrode. The other reduction peak at $+350 \mathrm{mV}$ is

\begin{tabular}{|c|c|c|c|}
\hline Drug & Description & $\begin{array}{c}\text { Reduction } \\
\text { potential }\end{array}$ & Reference \\
\hline $\begin{array}{c}\text { Cyclo- } \\
\text { phosphamide } \\
\text { Erythromycin }\end{array}$ & $\begin{array}{c}\text { Anticancer and } \\
\text { immunosuppressive }\end{array}$ & $-450 \mathrm{mV}$ & {$[12]$} \\
Ifosfamide & $\begin{array}{c}\text { Antibiotic } \\
\text { immunosuppressive }\end{array}$ & $-625 \mathrm{mV}$ & {$[13]$} \\
Indinavir & Anti-HIV & $-750 \mathrm{mV}$ & {$[12]$} \\
Verapamil & $\begin{array}{c}\text { For Hypertension, } \\
\text { angina pectoris, } \\
\text { cardiac arrhythmia }\end{array}$ & $-100 \mathrm{mV}$ & {$[15]$} \\
\multicolumn{2}{|c|}{} \\
\multicolumn{2}{|c|}{ Table 1- Reduction potential (corresponds to the current peak) for } \\
different drugs using Cytochrome P450 3A4
\end{tabular}




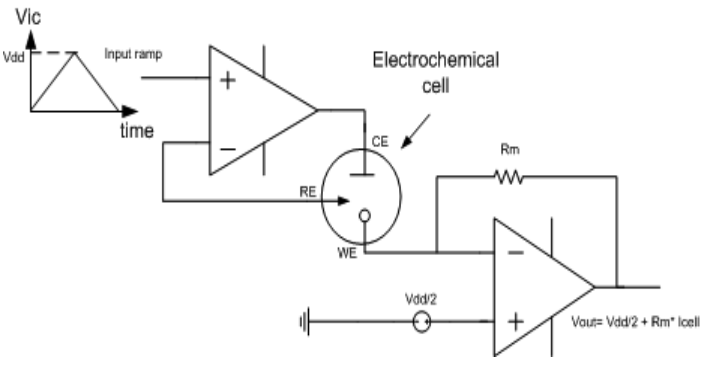

Fig 4. Electrochemical cell and a simplified potentiostat

probably due to the presence of secondary product that reacts on the working electrode when the reduction potential is applied and needs more investigations. The calibration curve worked out from the Gaussian approximation of the reduction peak at $+220 \mathrm{mV}$ in the cyclic voltammograms is illustrated in Fig. 3.

Detection limit and sensitivity are the parameters mostly used to evaluate the measurement quality. The calibration curve has a sensitivity of $7.3 \mathrm{uA} \mu \mathrm{M}^{-1} \mathrm{~cm}^{-2}$ (standard deviation of $0.65 \times 10^{-4}$ ), and a detection limit of $0.046 \mu \mathrm{M}$ (calculated as three-times the signal-noise ratio [16]). The detection limit is well below the pharmacological range. The measured current has a linear dependency to the drug concentration, which is compatible with the electrochemical theory [17]. This demonstrates the possibility of detecting Etoposide in its pharmacological range with a completely acceptable sensitivity and detection limit.

The obtained cyclic voltammograms in Fig. 2 show a $13 \mathrm{mV}$ potential shift in current peak position from the lowest to the highest Etoposide concentration. The shifts in the peak with increasing the drug concentration, shows the necessity of using a potential sweep to draw all the peaks corresponding to different concentrations.

On the other hand, Cytochrome P450 can be used for the construction of P450-based biosensor [8, 12] to detect different drugs presence in the electrochemical cell at the same time, using the fact that different drugs have their reduction peaks in different potentials that can be used as the drug's special fingerprint. So depending on what drugs are present in the solution, different peaks will appear in the cyclic voltammogram, Table 1 shows that reduction potential of some drugs detected by using Cytochrome P450 3A4 range from $-100 \mathrm{mV}$ to $-750 \mathrm{mV}$, which corresponds to the peak position in the cell current. Thus, for detecting and correctly

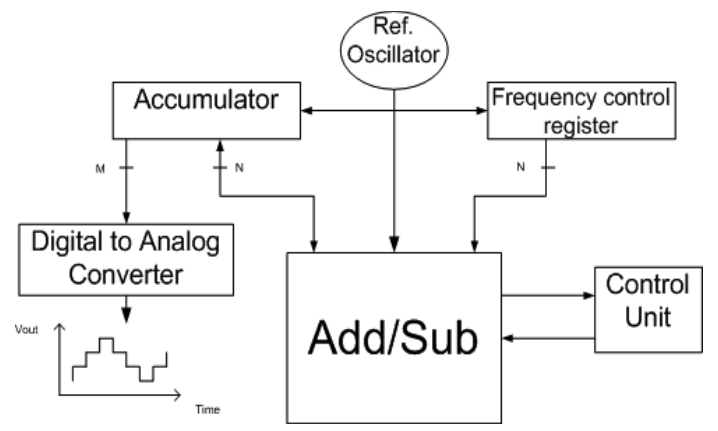

Fig. 6 DDS building blocks

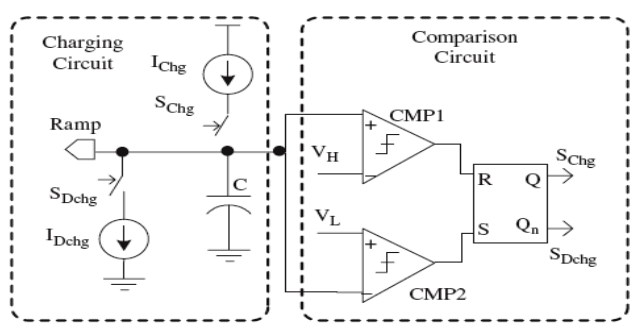

Fig 5. Capacitor charging/discharging method to generate the triangular voltage waveform

identifying different drugs within the electrochemical cell that is essential for multi- drug-detection, a linearly varying voltage needs to be applied to the electrochemical cell.

\section{CIRCUIT DESIGN}

Literature analysis of voltammetries with Etoposide and other drugs presented in [10] provided the following specifications for the circuit with three electrodes:

- To generate a triangular wave in amplitude range of -900 to $+900 \mathrm{mV}$ and scan rate 10 to $100 \mathrm{mV} / \mathrm{s}$.

- To read the current flowing through the solution in the range -1000 to $1000 \mu \mathrm{A}$.

In this work, we propose a method to generate the desired triangular voltage to enable the cyclic voltammetry measurement. To address the need for reading the current through the electrochemical cell, a circuit has been designed recently and presented in [6].

Fig. 4 shows a simplified electrochemical cell model with three electrodes together with two amplifiers acting as potentiostat and transimpedance amplifier. Potentiostat is used to apply and control the voltage between working and reference electrodes, and transimpedance amplifier is the most straightforward approach to convert the biosensor current into voltage for measurement purpose [18, 19].

\section{A. Circuit specifications}

To achieve the accurate voltammogram, the voltage between working and reference electrodes should change very slowly. If the voltage changes very rapidly, the biosensor current peak won't occur in the specific potential of the target molecule anymore. Therefore, it is not possible to distinguish among different targets. In the discussed case of

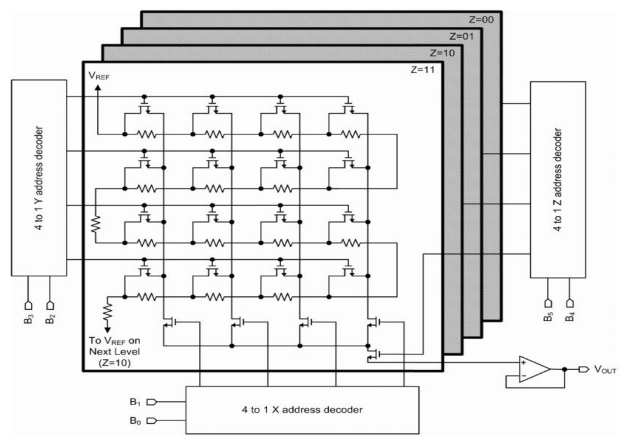

Fig. 7 Three dimension 6 bit DAC [21] 
Etoposide the best slope for the ramp, which leads to a good response, is about $20 \mathrm{mV} / \mathrm{sec}$ and the voltage ranges from $600 \mathrm{mV}$ to $+600 \mathrm{mV}$. To be able to measure cyclic voltammogram of different drugs such as in [10] with this generated ramp, a voltage range of $-900 \mathrm{mV}$ to $+900 \mathrm{mV}$ and a scan rate range of 10 to $100 \mathrm{mV} / \mathrm{s}$ are considered.

\section{B. Circuit architecture}

The conventional way to generate a ramp voltage is by charging and discharging a capacitor with a constant current source $[20,21,22]$. The resulting voltage across the capacitor is a ramp directly proportional with time,

$\mathrm{V}_{\text {out }}(\mathrm{t})=\mathrm{t} \times \mathrm{I}_{\mathrm{c}} / \mathrm{C}$

Fig. 5 shows the simplified circuit schematic of this method. Two comparators control the highest and lowest value of the triangular wave by turning off and on the current sources through the capacitor. In this circuit, the rising and falling slope of the generated ramp is directly proportional to the current sources value and inversely to the capacitor value. Assuming a maximum on chip capacitance about $100 \mathrm{pF}$, the required current to create this slope is few picoamperes. Current sources to generate this very low amount of current have not yet been achieved in any CMOS circuit, because it is in the range of MOS and capacitors leakage currents. So, providing an appropriate ramp potential that gives the correct peak is hardly possible by using analog design methods.

The method we are presenting here uses direct digital synthesizer (DDS) to generate a stepwise ramp voltage. It is a

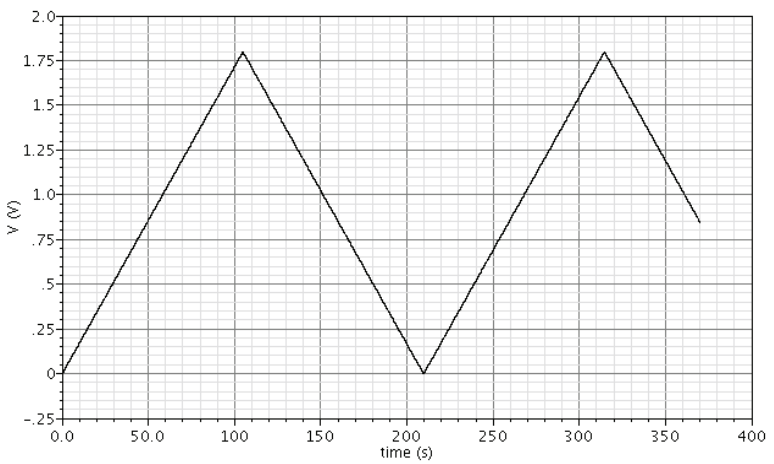

(a)

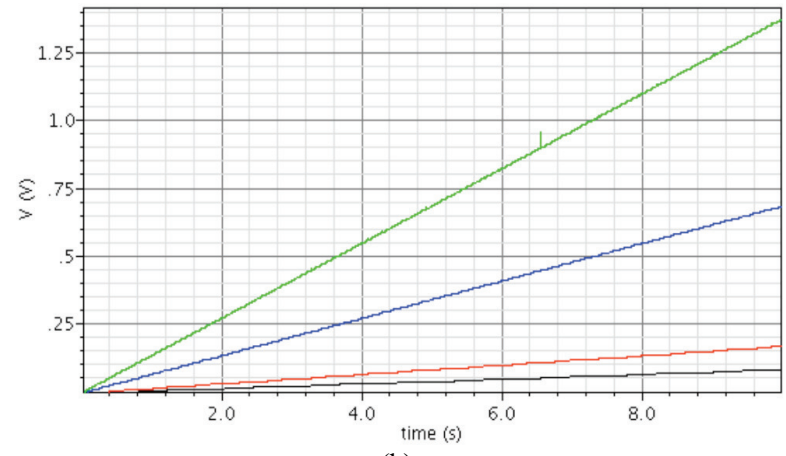

(b)

Fig. 8 Output voltage versus time, a) slope of the generated ramp is $17 \mathrm{mV} / \mathrm{sec}$. b) different scan rates $8.6,17,66 \& 133 \mathrm{mV} / \mathrm{sec}$ method to obtain timing signals from a clock source with programmable frequency. Generally a DDS waveform generator includes a numerically controlled oscillator and a digital to analog converter (DAC). The former produces at its output a discrete-time, quantized version of the desired output waveform whose period is controlled by the digital word contained in the frequency-control-register (FCR). The sampled, digital waveform is converted to an analog waveform by the $D A C$. Fig. 6 shows the block diagram of DDS designed to generate the triangular waveform.

An addition/subtraction block is used to add/subtract the FCR to/from the accumulator in every clock pulse. The FCR value determines the slope of the ramp. The FCR size and also the add/sub number of bits are carefully designed to provide different scan rates with different ramp slopes in the desired range. We chose the clock frequency to be $5 \mathrm{kHz}$ and considering 4 steps in the given scan rate of 10 to $100 \mathrm{mV} / \mathrm{s}$ to cover the needs in different voltammetric measurements. Register lengths and add/sub sizes are 19 bits. Add/Sub block is realized using 19 full adders.

The control unit is designed properly to generate the signals that are required for changing the operation from add to subtraction and vice versa, to provide the positive and negative slopes of the triangular wave. The last part of the circuit is the DAC. In this circuit the DAC will convert highest bits of the accumulator into analog waveform. The DAC accuracy is determined by the voltage resolution. In this application 9 MSB bits of accumulator are sufficient to yield the required resolution, which is $5 \mathrm{mV}$ in a full range of $1.8 \mathrm{~V}$.

Low power consumption is essential for the circuits, which are for implantable applications. Thus, the R-string structure

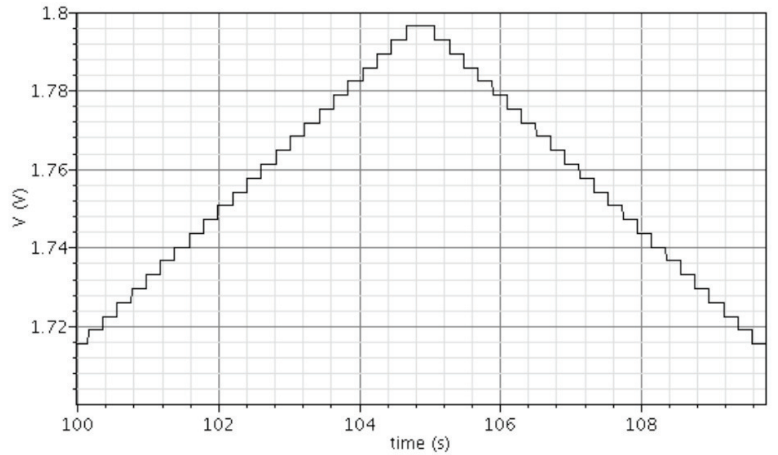

(a)

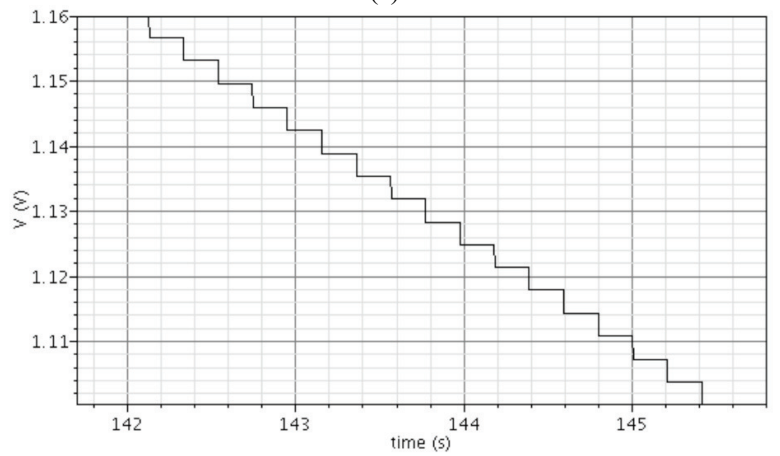

(b)

Fig. 9 Zoom in to the output voltage with the scan rate of $17 \mathrm{mV} / \mathrm{sec}$ a) in the peak of the voltage b) in decreasing part 
for DAC is selected [23]. A 9-bit DAC is realized using $2^{9}$ resistors (using undoped poly resistors). In this kind of DAC, analog multiplexers select the output voltage based on the digital input bits. In this work, three multiplexers are used, because according to [24] using three analog multiplexers to select the reference voltage leads to minimum number of logic gates for decoding the input signal and consequently lower delay, mismatch and area. Fig. 7 shows a threedimensional decoding scheme for a 6-bit DAC.

\section{Simulation RESUlts}

The circuit is implemented in 0.18um CMOS technology. Digital blocks of the developed architecture are implemented in VHDL, while the DAC circuit has been designed in Cadence Virtuoso. To reduce the simulation time, an output strobe is used with a period equal to $12 \times 10^{-6}$ times the generated ramp period. Fig. 8 shows the output voltage versus time for $8.6 \mathrm{mV} / \mathrm{sec}, 17 \mathrm{mV} / \mathrm{sec}, 66 \mathrm{mV} / \mathrm{sec}$ and $133 \mathrm{mV} / \mathrm{sec}$ scan rates. Fig. 9 shows precisely the stepwise ramp for the scan rate of the $17 \mathrm{mV} / \mathrm{sec}$ over a short time period. The average power consumption of the analog part of the circuit (i.e. DAC) is $300 \mathrm{nW}$, and the digital circuit consumes $400 \mathrm{nW}$.

\section{CONCLUSION}

In this paper the possibility of detecting a drug by cyclic voltammetry has been investigated. Electrochemical acquisitions of cyclic voltammograms on CNT-electrodes in presence of different concentration of drug have been shown. This study demonstrates the capability of cyclic voltammetry as a feasible technique for drug detection to implement in a biochip, for improving the biological recognition.

A circuit schematic to generate a very low slope and low frequency ramp voltage to measure the cyclic voltammetry has been proposed based on the DDS method. The circuit has been designed to encompass more drugs detection using $\mathrm{CV}$ method in implantable applications and it is implemented in 0.18 um technology. The slope of the generated ramp voltage is controllable and varies from less than $10 \mathrm{mV} / \mathrm{sec}$ to more than $100 \mathrm{mV} / \mathrm{sec}$ with a rail-to-rail swing. The power consumption of the whole circuit consisting of both analog and digital parts is $700 \mathrm{nW}$. The low power consumption makes this circuit also suitable for applications with fully implantable and remotely powered devices [25]. The presented circuit together with a current readout circuit will be fabricated on a chip to enable drug detection based on the $\mathrm{CV}$ method in a three-electrode biosensor cell.

\section{ACKNOWLEDGMENT}

The research has been funded by the i-IronIC project that is financed with a grant from the Swiss Nano-Tera.ch initiative and evaluated by the Swiss National Science Foundation; the research has also been funded by the SNF Sinergia project (code CRSII2- 127547/1).

\section{REFERENCES}

[1] B. B. Spear, M. Heath-Chiozzi and J. Huff, "Clinical application of pharmacogenetics", Trends in Molecular Medicine, vol.7, pp.201-206, 2001.

[2] G. N. Hortobagyi, "Treatment of breast cancer", New England Journal of Medicine, vol.339, pp.974-984, 1998.

[3] A.Y. Chang, L. Hui, R. Asbury, L. Boros, G. Garrow, and J. Rubins, "Ifosfamide, carboplatin and etoposide (ICE) in metastatic and refractory breast cancer", Cancer Chemother Pharmacol, vol.44, pp.526-528, 1999.

[4] N. Gad-El-Mawla, "Use of ifosfamide in the management of breast cancer", Annals of Oncology, vol.3, pp.S21-S23, 1992.

[5] S. Carrara, V. V. Shumyantseva, A. I. Archakov, and B. Samor1, "Screen-printed electrodes based on carbon nanotubes and cytochrome P450scc for highly sensitive cholesterol biosensors", Biosensors and Bioelectronics journal, vol.24, pp.148-150, 2008.

[6] S. Carrara, M. D. Torre, A. Cavallini, D. De Venuto, and G. De Micheli, "Multiplexing $\mathrm{pH}$ and Temperature in a Molecular Biosensor", Proceedings of the IEEE BIOCAS Conference, , pp.146-149, 2010.

[7] K. R. HÃnde,P. J. Wedlund, R. M. Noone, G. R. Wilkinson, F. Anthony Greco, and S. N. Wolff, "Pharmacokinetics of High-Dose Etoposide (VP-16-213) Administered to Cancer Patients", Cancer Research, vol.44, pp.379-382, 1984.

[8] S. Carrara, A. Cavallini, A. Garg, and G. De Micheli , "Dynamical spot queries to improve specificity in P450s based multi-drugs monitoring," International Conference on Complex Medical Engineering ICME, pp.1-6, 2009.

[9] C. E. Banks, Trevor J. Davies, Gregory G. Wildgoose and Richard G. Compton, "Electrocatalysis at graphite and carbon nanotube modified electrodes: edge-plane sites and tube ends are the reactive sites", Chem. Commun., pp.829-841, 2005.

[10] S. Carrara, A. Cavallini,G. De Micheli, J. Olivo, L. Benini, V. V. Shumyantseva, and A. I. Archakov, "Circuits Design and NanoStructured Electrodes for Drugs Monitoring in Personalized Therapy", Conference Proceedings of IEEE BioCAS, 2008.

[11] J.J.M. Holthuis, W.J. van Oort, F.M.G.M. Romkens, J.Renema, and P. Zuman, "Electrochemistry of podophyllotoxin derivatives: Part I. Oxidation mechanism of etoposide (VP 16-213)", Journal of Electroanalytical Chemistry, vol.220, pp101-124, 1987.

[12] C. Baj-Rossi, G. De Micheli, and S. Carrara, "P-450-Based nanobiosensors for personalized medicine", Invited book's chapter in A. Serra (Ed) "Biosensors for Health, Environment and Biosecurity", ISBN: 978-953-307-155-8, InTech Publisher, Vienna, 2011, in press.

[13] N. R. Hendricks, T. T.Waryo, O. Arotiba, N. Jahed, P. G.L. Baker, and E. I. Iwuoha, "Microsomal cytochrome P450-3A4 (CYP3A4) nanobiosensor for the determination of 2,4-dichlorophenol-An endocrine disruptor compound", Electrochimica Acta [0013-4686] Hendricks, vol:54, pg:1925, 2009.

[14] A. Ignaszak, N. Hendricks, T.Waryo, E. Songa, N. Jahed, R. Ngece, A. Al-Ahmed, B. Kgarebe, P. Baker, and E.I. Iwuoha, "Novel therapeutic biosensor for indinavir-A protease inhibitor antiretroviral Drug", Journal of Pharmaceutical and Biomedical Analysis, vol.49, pp.498501,2009

[15] S. Joseph, J. F. Rusling, Y. M. Lvov, T. Friedberg, and U. Fuhr, "An amperometric biosensor with human CYP3A4 as a novel drug screening tool", Biochemical Pharmacology, vol.65, pp. 1817-1826, 2003.

[16] I. Lavagnini, R. Antiochia, and F. Magno, "A Calibration-Base Method for the Evaluation of the Detection Limit of an Electrochemical Biosensor", Electroanalysis, vol.19, pp.1227-1230, 2007.

[17] A. Bard and L. Faulkner, "Electrochemical methods: fundamentals and applications", Wiley New York, 1980.

[18] M. Ahmadi and G. Jullien, "Current-mirror-based potentiostats for three-electrode amperometric electrochemical sensors", IEEE Transactions on Circuits and Systems Part I: Regular Papers, vol. 56, pp. 1339-1348, 2009. 
[19] ". Narula and J. Harris, "A time-based VLSI potentiostat for ion current measurements," IEEE Sensors Journal, vol. 6, pp. 239-247, 2006.

[20] F. azais, S. Bernard, Y. Bertrand, X. Michel, and M. Renovell, "a low cost adaptive ramp generator for analog BIST applications", $19^{\text {th }}$ IEEE proceeding on. VTS, 2001.

[21] J.Wang, E Sanchez-Sinencio and F. Maloberti, "Very linear rampgenerators for high ADC BIST and calibration", IEEE midwest symp On circuits and systems, 2000.
[22] H.P Forghani-zadeh and G.A. Rincon-Mora, "Low power CMOS ramp generator circuit for DC-DC converters", journal of low power electronics, vol.2, pp 1-5, 2006.

[23] B. Razavi, Principles of data conversion system design, IEEE Press, 1995

[24] I. Knausz and R.J. Bowman, "A low power, scalable, DAc architecture for liquid crystal display drivers", IEEE journal of solid state circuits, vol.44, pp.2402-2410, 2009

[25] J. Olivo, S. Carrara, and G. De Micheli, "Energy Harvesting and Remote Powering for Implantable Biosensors", IEEE Sensors Journal, In press. 